\title{
Seelen ohne Heimat: Zur Situation von Asylsuchenden in Deutschland
}

\author{
Souls without a Home: The Situation of Asylum Seekers in Germany
}

Autoren

Institute
Marcel Sieberer $^{1,2}$, Wielant Machleidt ${ }^{1}$

Klinik für Psychiatrie, Sozialpsychiatrie und Psychotherapie, Medizinische Hochschule Hannover

Ameos Klinikum Hildesheim, Fachklinikum für Psychiatrie und Psychosomatik

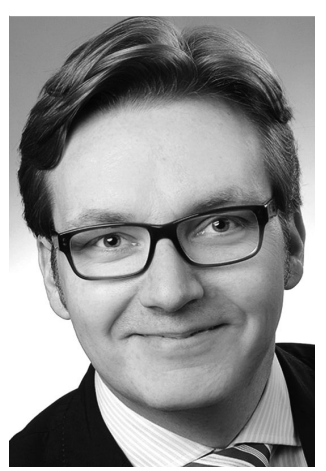

Marcel Sieberer

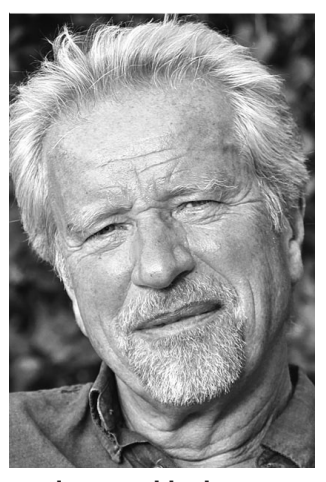

Wielant Machleidt

Bibliografie

DOI http://dx.doi.org/

$10.1055 / \mathrm{s}-0034-1387643$

Psychiat Prax 2015; 42:

175-177

(c) Georg Thieme Verlag KG Stuttgart · New York

ISSN 0303-4259

Korrespondenzadresse

Priv.-Doz. Dr. med.

Marcel Sieberer

Ärztlicher Direktor des Ameos Klinikum Hildesheim Goslarsche Landstraße 60 31135 Hildesheim

msib.psy@hildesheim.ameos.de

\section{Steigende Asylantragszahlen \\ $\nabla$}

Im vergangenen Jahr hat sich der seit 2008 abzeichnende Trend eines wieder wachsenden $\mathrm{Zu}$ stroms von Asylsuchenden nach Deutschland fortgesetzt und augenscheinlich verstärkt. Von den über 200000 im Jahr 2014 in Deutschland gestellten Asylanträgen handelte es sich in über 85\% um Erstanträge auf Asyl, was im Vergleich zum Vorjahr einen Anstieg um rund 58\% bedeutet. Gemessen an der Gesamtzahl der gestellten Asylanträge handelte es sich beim Jahr 2014 um das Jahr mit den vierthöchsten Antragszahlen seit Bestehen der Bundesrepublik. Bisher sind nur in den Jahren 1991 - 1993 mehr Asylanträge verzeichnet worden, wobei alleine in 1993 knapp 440000 Erst- und Folgeanträge auf Asyl gestellt wurden. Anhand der Statistik des Bundesamts für Migration und Flüchtlinge lässt sich seit den 1970erJahren ein wellenartiger Verlauf der Asylantragszahlen nachvollziehen. Dabei wechseln auch die zugangsstärksten Herkunftsländer der Asylsuchenden, was im Wesentlichen die jeweilige politische Situation in Europa und in der Weltgemeinschaft reflektiert. Im Jahr 2014 kam annähernd ein Viertel aller Asylerstantragsteller aus Syrien [1]. Knapp 10\% aller Asylerstanträge stellten im gleichen Jahr Menschen aus Serbien, gefolgt von Eritrea (7,6\%) und Afghanistan (5,3\%). Wenn sich die jüngste Entwicklung der Asylantragszahlen fortsetzt, werden am Ende des laufenden Jahres nochmal deutlich mehr Asylsuchende in Deutschland Zuflucht gesucht haben als in 2014. Da die Aufnahme von Asylsuchenden bereits 2014 die Länder, Städte und Kommunen in Deutschland vor zum Teil erhebliche Probleme gestellt hat, erscheinen die offenen Fragen nach einer angemessenen Unterbringung, den Möglichkeiten einer Integration oder der gesundheitlichen Versorgung von Asylsuchenden gegenwärtig umso drängender.

\section{Psychodynamik der Fremdenfeindlichkeit $\nabla$}

Unter dem Eindruck eines wachsenden Zustroms von Asylsuchenden hat sich im vergangenen Jahr der öffentlich geführte Diskurs um das Thema Zuwanderung verschärft. Nicht zuletzt hat die Formierung der Pegida-Bewegung, die ab Herbst 2014 in einigen Städten Deutschlands durch regelmäßige Demonstrationen und Kundgebungen sichtbar wurde, zu einer Polarisierung der Meinungsbildung und Debatte über die gegenwärtige Einwanderungs- und Asylpolitik beigetragen. Gleichzeitig haben sich als Reaktion auf die fremdenfeindlichen Strömungen vielerorts aber auch Bürger zu Gegenbewegungen zusammengeschlossen und für mehr Toleranz, Offenheit und Integration eingesetzt. Die Menschen, die hierzulande Asyl suchen, sind damit gewissermaßen zu einer Projektionsfläche für gegensätzliche Auffassungen, Bewertungen und Emotionen geworden, die in der Gesellschaft zu den Themen Zuwanderung, kulturelle oder nationale Identität und Integration bestehen. Die Hintergründe und die Entwicklung solcher Phänomene wie Pegida sind durch klassische Deutungsmuster und Erklärungstheorien der Soziologie zu Ursachen von Vorurteilen und Feindbildern nicht mehr erschöpfend zu erklären. So scheint sich eine ablehnende Haltung zum Thema Zuwanderung hierzulande längst nicht mehr nur auf die Teile der Gesellschaft zu beschränken, die selbst von sozioökonomischen Nachteilen betroffen sind und sich deshalb mit Zuwanderern in einer vermeintlichen Konkurrenzsituation um knappe und begehrte Ressourcen sehen. Vielmehr mischt sich mit vordergründig rationalen, ökonomischen und primär individualpsychologisch verankerten Gründen offensichtlich ein triebhaft-irrationales Moment [2], dessen Erklärung eher in gruppenpsychologischen Phänomenen zu suchen ist. Ethologisch ist die Furcht vor dem Fremden und dessen aggressive Abwehr zunächst als eine ent- 
wicklungsgeschichtlich tief verwurzelte, archaische Reaktionsform zu verstehen, die vorzivilatorisch angelegt und der Organisation und Abgrenzung von Gruppenverbänden geschuldet ist (vgl. [2]). In der Tradition entwicklungspsychologischer Theorien der Psychoanalyse wurde in jüngerer Zeit mehr der ambivalente Charakter der Angst vor dem Fremden herausgearbeitet, die zwischen Furcht auf der einen Seite und Neugier oder gar Lust auf der anderen Seite zu oszillieren scheint. Nach Erdheim (1992 [2]) bildet sich neben einer Selbst- und Objektrepräsentanz zusätzlich eine Fremdenrepräsentanz aus, die die Grundlage für eine Beziehung zu einer Person darstellt, die eben nicht die Mutter ist (zitiert nach [2]). Durch projektive Abwehrmechanismen besteht dabei das Risiko, dass die Fremdenrepräsentanz zum Ort externalisierter, abgelehnter bzw. gefürchteter und gleichzeitig gehasster Selbstanteile wird. Kurz gesagt wird dadurch das Eigene zum Guten und das Fremde zum Bösen [3].

Das Phänomen der Fremdenfeindlichkeit kann in dieser Lesart mithin als ein Symptom verstanden werden, das etwas über den psychosozialen Zustand einer Gesellschaft aussagt. Die Ursachen und Auswirkungen von Fremdenfeindlichkeit müssen entsprechend genauer analysiert und verstanden werden, um das hinsichtlich der Zivilgesellschaft selbstdestruktive Potenzial besser erkennen und begrenzen zu können.

\section{Psychische Gesundheit Asylsuchender \\ $\nabla$}

Trotz der bereits lange bestehenden Relevanz für das öffentliche Gesundheitswesen gibt es bisher keine systematischen wissenschaftlichen Untersuchungen über den Gesundheitszustand und zur Gesundheitsversorgung von Asylsuchenden in Deutschland [4]. In den wenigen systematischen Reviews zur Prävalenz psychischer Störungen bei Asylsuchenden in westlichen Industrieländern wurden daher Untersuchungen aus Deutschland bisher auch nicht berücksichtigt (vgl. [5, 6]). Zur Prävalenz psychischer Störungen bei Asylsuchenden sind selbst international nur wenige Untersuchungen durchgeführt worden. Die Prävalenzraten für psychische Störungen bei Asylsuchenden und Flüchtlingen variieren in diesen Studien ganz erheblich, beispielsweise für Depressionen von unter $3 \%$ bis $80 \%$ oder für Posttraumatische Belastungsstörungen (PTBS) von 4,4\% bis $86 \%$ [7]. Diese hohe Variabilität wird zum Teil auf methodische Unterschiede der Studien zurückgeführt, könnte gleichzeitig aber auch die unterschiedlichen Bedingungen widerspiegeln, auf die Asylsuchende in den jeweiligen Ankunftsländern treffen. Insgesamt sprechen die Studienergebnisse aus westlichen Industrieländern dafür, dass Flüchtlinge und Asylsuchende nahezu doppelt so häufig von Depressionen oder Angststörungen betroffen sind wie sogenannte Arbeitsmigranten, und zwar unabhängig von den ökonomischen Rahmenbedingungen der Aufnahmeländer [5]. Zudem hat eine Reihe von Untersuchungen bestätigt, dass Asylsuchende im Vergleich zur Mehrheitsbevölkerung oder zu anderen Migrantengruppen deutlich häufiger von PTBS betroffen sind [6, 8]. Korrespondierend sind für Asylsuchende neben hohen Raten von prämigratorischen Traumatisierungen eine Reihe charakteristischer postmigratorischer Stressoren identifizierbar, die zur Manifestierung oder zur Chronifizierung psychischer Störungen beitragen können. In diesem Zusammenhang werden bspw. der unsichere Aufenthaltsstatus, Widrigkeiten des Asylverfahrens, Erfahrungen von Diskriminierung und Vorurteilen, fehlende Arbeitsmöglichkeiten und der erschwerte Zugang zur Gesundheitsversorgung genannt [9-11]. Gerade diese Umstände kennzeichnen aber vie- lerorts die Bedingungen, unter denen Asylsuchende gegenwärtig in Deutschland leben.

\section{Gesundheitsversorgung und psychosoziale Forschung $\nabla$}

Ein strukturelles Hauptproblem bei der medizinischen Versorgung von Asylsuchenden liegt in den Limitationen, die das Asylbewerberleistungsgesetz (AsylbLG) vorgibt. In dem aus politischer Perspektive und seitens medizinischer Fach- und Sozialverbände umstrittenen Regelungswerk ist festgelegt, dass sich die medizinische Versorgung von Asylsuchenden ausschließlich auf akute Erkrankungen, Schmerzzustände oder lebensbedrohliche Gesundheitsstörungen zu beschränken hat. Auf diesem Hintergrund ist es für Asylsuchende besonders schwierig, eine angemessene psychiatrisch-psychotherapeutische Behandlung zu erhalten. Die Bundespsychotherapeutenkammer fordert bereits seit Längerem, bundesweit einheitliche Regelungen zu schaffen, nach denen Asylsuchende im Bedarfsfall einen Zugang zum psychiatrisch-psychotherapeutischen Versorgungssystem erlangen können. Das Risiko der gegenwärtigen Regelungspraxis besteht darin, dass ein großer Anteil der Asylsuchenden mit schweren psychischen Störungen, insbesondere mit Traumafolgestörungen, ohne Aussicht auf eine angemessene psychiatrische und insbesondere psychotherapeutische Behandlung bleibt und somit psychisches Leid potenziell noch vergrößert wird. Die DGPPN hat Ende des vergangenen Jahres deshalb nochmals gefordert, durch entsprechende Maßnahmen die psychiatrisch-psychotherapeutische Versorgung von Flüchtlingen und Asylsuchenden in Deutschland zu verbessern [12]. Neben dem Aufbau einer interkulturellen Kompetenz im Versorgungssystem wurde ausdrücklich die gesetzliche Regelung einer Kostenübernahme von Sprachvermittlern im Zusammenhang mit Gesundheitsleistungen angemahnt. Zudem wurde eine bundesweite Einführung gestufter Behandlungsmodelle gefordert, da bestehende Versorgungsstrukturen bereits in der Vergangenheit nicht ausreichten, um eine adäquate medizinische und vor allem eine psychiatrisch-psychotherapeutische Versorgung dieser benachteiligten Patientengruppe zu gewährleisten.

Durch die Einführung der Gesundheitskarte für Asylsuchende soll aktuell in ersten Bundesländern, namentlich in Bremen und Hamburg, der Zugang zu Gesundheitsleistungen erleichtert werden. Ob sich dadurch die Behandlungsmöglichkeiten auch für psychische Störungen in der Praxis verbessern werden, bleibt zumindest zweifelhaft, da nicht nur administrative Hürden, sondern im Einzelfall auch kulturelle und sprachliche Barrieren überbrückt werden müssen. Da in vielen Regionen Deutschlands die psychotherapeutische Versorgung grundsätzlich schon mit mehrmonatigen Wartezeiten auf einen Therapieplatz verbunden ist, ist zu befürchten, dass die Zugangsbarrieren für Asylsuchende ungleich höher bleiben, zumal sich durch die Einführung einer Gesundheitskarte die einschränkenden Vorgaben des AsylbLG inhaltlich nicht ändern.

Ohne Frage gehört Deutschland in Europa zu den bevorzugten Zielländern von Asylsuchenden und Flüchtlingen. Der gegenwärtig wieder wachsende Zustrom stellt nicht nur die Länder, Städte und Kommunen vor enorme Probleme, sondern konfrontiert auch das Gesundheitssystem mit neuen Herausforderungen und offenen Fragen, z. B. dazu wie eine angemessene Gesundheitsversorgung für eine zunehmende Anzahl von Asylsuchenden aus unterschiedlichsten Herkunftsländern und unter den genannten Bedingungen gewährleistet werden kann. Da bisher in Deutsch- 
land keine Routinedaten zur gesundheitlichen Situation und zur Gesundheitsversorgung der überaus heterogenen Gruppe der Asylsuchenden vorliegen, ist die Entwicklung innovativer, sachund bedarfsgerechter Versorgungsstrukturen zusätzlich erschwert. Notwendig sind daher förderliche Rahmenbedingungen für interdisziplinäre Forschungsansätze, in denen die Situation von Asylsuchenden in Deutschland aus unterschiedlichen wissenschaftlichen Perspektiven beleuchtet wird und deren Ergebnisse eine fundierte Grundlage für die Asyldebatte liefern könnten. In jedem Fall bedarf es dringend neuer Signale von Akteuren aus der Politik, der Zivilgesellschaft und der Sozialsysteme, die gleich auf mehreren Ebenen durchgreifende Nachbesserungen der bestehenden Konzepte zur Aufnahme, Integration und vor allem auch der Gesundheitsversorgung von Asylsuchenden initiieren.

\section{Literatur}

1 Bundesamt für Migration und Flüchtlinge (BAMF). Schlüsselzahlen Asyl 2014. Im Internet: http://www.bamf.de/SharedDocs/Anlagen/ DE/Publikationen/Flyer/flyer-schluesselzahlen-asyl-jahr-2014.html (Stand: 20.03.2015)

2 Wirth $\mathrm{H}-\mathrm{J}$. Fremdenhass und Gewalt als familiäre und psychosoziale Krankheit. Psyche 2001; 11: 1217-1244

3 Erdheim M. Das Eigene und das Fremde. Über ethnische Identität. In: Jansen MM, Prokop U, Hrsg. Fremdenangst und Fremdenfeindlichkeit. Basel: Stroemfeld; 1993: 163-182
4 Schneider C, Mohsenpour A, Joos S et al. Health status of and health-care provision to asylum seekers in Germany: protocol for a systematic review and evidence mapping of empirical studies. Syst Rev 2014; 3: 139

5 Lindert J, Ehrenstein OS, Priebe $S$ et al. Depression and anxiety in labor migrants and refugees - a systematic review and meta-analysis. Soc Sci Med 2009; 69: 246-257

6 Fazel M, Wheeler J, Danesh J. Prevalence of serious mental disorder in 7000 refugees resettled in western countries: a systematic review. Lancet 2005; 365: 1309-1314

7 Bogic M, Ajdukovic D, Bremner $S$ et al. Factors associated with mental disorders in long-settled war refugees: refugees from the former Yugoslavia in Germany, Italy and the UK. Br J Psychiatry 2012; 200: $216-223$

8 Gäbel U, Ruf M, Schauer M et al. Prävalenz der Posttraumatischen Belastungsstörung [PTSD] und Möglichkeiten der Ermittlung in der Asylverfahrenspraxis. Z Klin Psychol Psychother 2006; 35: 12 -20

9 Knipscheer JW, Kleber RJ. The relative contribution of posttraumatic and acculturative stress to subjective mental health among Bosnian refugees. J Clin Psychol 2006; 62: 339-353

10 Robjant $K$, Hassan R, Katona C. Mental health implications of detaining asylum seekers: systematic review. Br J Psychiatry 2009; 194: $306-$ 312

11 Pumariega AJ, Rothe E, Pumariega JB. Mental health of immigrants and refugees. Community Ment Health J 2005; 41: 581 - 597

12 Deutsche Gesellschaft für Psychiatrie und Psychotherapie, Psychosomatik und Nervenheilkunde (DGGPN). Traumatisierte Kriegsopfer: psychiatrisch-psychotherapeutische Hilfsangebote ausbauen. Presseinformation Nr. 37 (27.11.2014). Im Internet: http://www.dgppn.de/ fileadmin/user_upload/_medien/download/pdf/pressemitteilungen/ 2014/2014-11-27-DGPPN-Pressemitteilung_Traumatisierungen.pdf (Stand: 18.3.2015) 